\title{
Microcontroller Based Electrical Self-Test and Self-Calibration of MOX Sensor Systems
}

\author{
M.Stahl-Offergeld ${ }^{1}$, Hans-Peter Hohe ${ }^{1}$, D. Krause ${ }^{1}$, M. Gorny ${ }^{1}$ \\ ${ }^{1}$ Fraunhofer Institute for Integrated Circuits IIS, Am Wolfsmantel 33, 91058 Erlangen, Germany \\ Markus.Stahl-Offergeld@iis.fraunhofer.de
}

\begin{abstract}
:
CMOS integrated sensor interfaces offer high performance on a small area. In general, CMOS processes have very good matching between the devices on chip, but absolute values can vary about more than $30 \%$. For absolute measurements, it is necessary to calibrate every single chip. The sensor interface for MOX gas sensors, which was designed inside the FP7 project MSP, thus offers several self-test and self-calibration building blocks to reduce the calibration effort. Together with the microcontroller of the sensor system, it is possible to do the complete self-test and self-calibration of the system without additional measurement equipment.
\end{abstract}

Key words: MOX Sensor System, Self-Test, Self-Calibration, Sensor Interface

\section{MOX sensor system for temperature cycled operation}

The temperature cycled operation (TCO) of MOX gas sensors makes it possible to measure VOCs in the range of a few ppb [1]. The CMOS integrated MOX sensor interface presented in [2] offers all relevant circuits to fulfill the high demands.

CMOS processes allow the integration of complex circuits on a small area. They combine high performance with low power consumption, but need to be calibrated to perform accurate measurements. To demonstrate the complexity of the calibration itself, the ASIC is presented first.

The MSP ASIC has a register based SPI interface working with up to $16 \mathrm{MHz}$. It is possible to configure the interface, do all necessary measurements and implement a TCO working with $10 \mathrm{kHz}$. To operate all kinds of MOX sensors, the heater is controlled either in constant current ( $5 \mathrm{~mA}$ to $40 \mathrm{~mA}$ ) or in constant voltage $(5 \mathrm{~V}$ or $20 \mathrm{~V})$ mode. While setting the current, the voltage across the heater is measureable at the same time and vice versa.

For resistance measurement of the sensitive layer, the bias voltage across the sensor can be configured to a value between $0 \mathrm{~V}$ and $1.5 \mathrm{~V}$ while an internal shunt enables sensor current observation. The voltage across the shunt is amplified with a programmable gain amplifier (gain 1 to 4096) for a measurement range of $100 \Omega$ to $100 \mathrm{M} \Omega$. Two integrated delta sigma analog to digital converters allow for parallel measurements of the heater and sensitive layer.

For high accuracy measurements, it is necessary to calibrate all analog building blocks. The bandgap voltage is trimmed to $1.18 \mathrm{~V}$ first, as this is the reference voltage for all analog building blocks on the chip. Now the reference-voltages and -currents are stable over a wide temperature range. The next step is the calibration of the heater current source and the heater voltage measurement path. Finally the voltage sources, which set the voltage across the sensitive layer, the current measurement path and the gains of the amplifier, have to be calibrated, too. All this has to be done with the final sensor system.

\section{Design for self-testability}

The test- and calibration-time of sensor systems is a cost factor not to neglect. That is why the design for self-testability should be an important requirement in all ASIC designs. In the MSP ASIC, a lot of test modes have been implemented. Core of the test concept is a test bus. It can connect all important internal nodes to dedicated test pins. With this pins it is possible to test the analog building blocks. These tests can be done in a test house so only working devices will be assembled on the PCB. 
Additionally, this test bus enables selfcalibration on module level with just one additional $10 \mathrm{k} \Omega$ resistor (RCali) which has to be connected to the test bus. This resistor can be part of the sensor system and must not be removed for normal application.

\section{Self-Test and Self-Calibration}

To demonstrate the working principle of the test concept, fig. 1 shows a detail of the test concept.

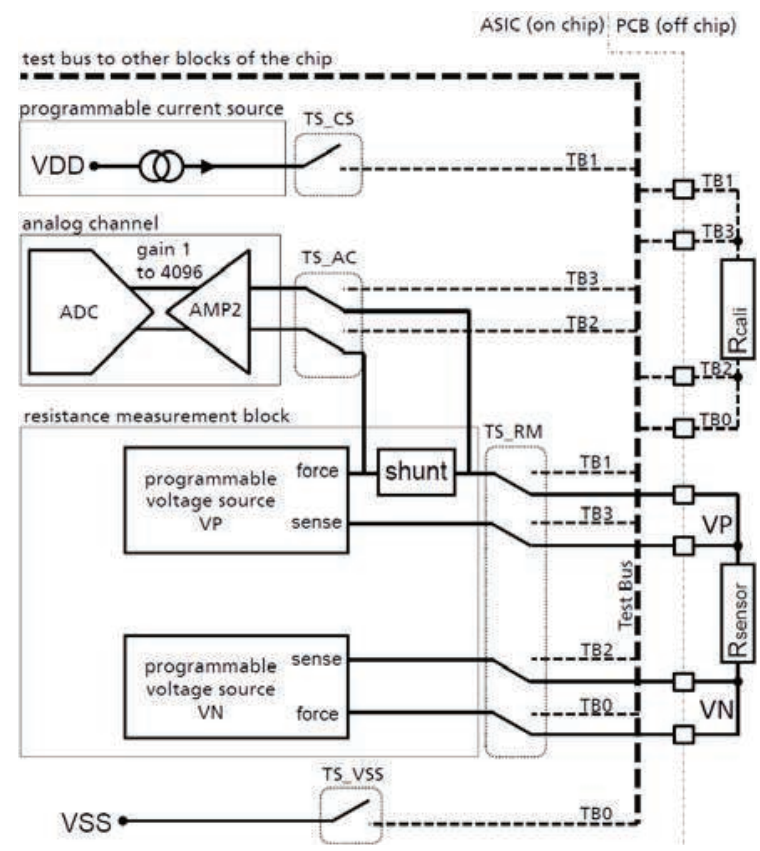

Fig. 1. Test concept to measure the gains of the analog channel, and to calibrate the resistance measurement block.

Gain self-calibration: Using test switches TS_CS and TS_VSS the integrated programmable current DAC (IDAC), which also can be used to measure the sensor resistance (not shown in fig. 1), is connected to TB1 while TBO is connected to VSS. With an IDAC current of $50 \mu \mathrm{A}$ a test voltage of $0.5 \mathrm{~V}$ is generated across RCali. Using gain 1 a signal of $0.5 \mathrm{~V}$ is digitized. Setting the gain to 2 increases the input voltage of the ADC to $1 \mathrm{~V}$. The ratio of both digital measured signals equals the ratio between gain 1 and 2 . Now the Current is set to $20 \mu \mathrm{A}$, which enables the measurements with gain 2 and 4. Again, the ratio of the digital measurements equals the ratio between gain 2 and 4 . This sequence continues until gain 4096 . The absolute gain values might not be exact, but this is not important for the sensor resistance measurement. The matching between the gains is much more crucial as a switch from one gain to another should not result in a step in the measured signal.

Resistance measurement self-calibration: To calibrate the resistance measurement block it is possible to disconnect the building block from the sensor and connect it to the test bus with the known $10 \mathrm{k} \Omega$ resistor using test switches TS_RM. Now the resistance measurement is aligned to RCali, including the calibrated gain setting of the PGA and the configured bias voltage (VP-VN) across the sensor. In the next step the bias voltage across the sensor (VPVN) can be calibrated by changing the voltage across the sensor and calculate the impact between these two measurements. Again, the absolute value of the bias voltage is not important. Only the matching between the voltage steps has an impact on the resistance measurement.

Analog channel self-calibration: If the analog channel should be used as an absolute voltage meter, the absolute value needs to be calibrated. The used microcontroller in the sensor system has several ADC inputs, which can be used. If the supply voltage is known exactly during the self-test, it can be used as reference voltage for the ADC of the $\mu \mathrm{C}$. Otherwise it is possible to use a discrete reference voltage chip in the sensor system. These reference voltages are very exact and enable self-tests during the lifetime of the system.

\section{Conclusion}

The integrated building blocks for self-test and self-calibration allow for a complete electrically self-calibration on module level, using a microcontroller and one additional resistor which can be integrated into the sensor system on PCB level. This way, a fast and easy calibration of the sensor system is implemented. Additionally, these routines can be used during the lifetime of the system to monitor the system performance.

\section{Acknowledgements}

This work has received funding from the European Community's FP7-ICT-2013-10, MSP- Multi-Sensor-Platform for Smart Building Management under the project $n^{\circ} 611887$

\section{References}

[1] M. Leidinger, T. Sauerwald, W. Reimringer, G. Ventura, A. Schütze, Selective detection of hazardous VOCs for indoor air quality applications using a virtual gas sensor array, J. Sens. Sens. Syst. 3, 253-263 (2014)

[2] M. Stahl-Offergeld, H.-P. Hohe, M. Hackner, Low Power High Speed CMOS Interface for MOS Gas Sensors, Procedia Engineering 120, 1046-1049 (2015) 